\title{
Gleichmäßige asymptotische Darstellungen für Parameterintegrale mit zwei reellen Parametern ${ }^{1}$ )
}

\author{
H.-J. SCHELL
}

Für eine Klasse von Integralen, die von zwei reèllen Parametern $s, \alpha$ abhängen, werden für eine Beweguing $s \rightarrow s_{0}$ mehrere asymptotische Darstellungen hergeleitet; die. gleichmäßig für alle $\alpha$ aus einem gewissen Intervall gelten: Dabei werden zwei Fälle unterschieden: a) der Integrand hat ein einfaches Maximum im Innern des Integrationsintervalles; b) der Integrand hat keine stationäre Stelle im Integrationsinterväll. In beiden Fällen gelten die Resultate auch noch, wenn die stationäre Stelle mit der unteren Integrationsgrenze zusammenfällt.

Для интегралов, зависящих от двух действительных параметров s и $\alpha$, выводятся асимптотические равенства, имеющие место для случая $s \rightarrow s_{0}$ и равномерно для всех $\alpha$ из некоторого отрезка. Рассмотрепы два случая: а) полинтегральная функция имеет простой максимум внутри отрезка пнтегрирования; б) она не имеет стационарной точки внутри отрезка пнтегрирования.' В обеих случаях результат остаетсл справеаливым, если стационарная точка совпадает с нижним пределом йнтегрирования.

Several asymptotic approximations, valid uniformly on certain $\alpha$-intervals, are obtained for a class of integrals depending on two real parameters $s$ and $\alpha$, where $s$ tends to $s_{0}$. Two cases are considered: a) the integrand has a simple maximum interior to the interval of integration, b) the interval of integration contains no stationary point. In both cases the results still hold true if the stationary point coalesces with the lower limit of integration.

\section{Einleitung}

Die in dieser Arbeit betrachteten Parameterintegrale haben die Form

$$
F(s, \alpha)=\int_{x(s, \alpha)}^{o b(s, \alpha)} e^{-g(s, \alpha, t)} d t .
$$

Es sei $g(s ; \alpha, t)$ für alle $(s, \alpha, t) \in S \times A \times[x, b)$ definiert; wobei $S$ eine Menge reeller Zahlen und $A$ ein Intervall sei, das von $s$ abhängen darf; es ist auch $b=\infty$ zugelassen, jedoch soll $x$ endlich sein, und das Integral in (1) sei konvergent. Für die genannten $(s, \alpha, t)$ sei $g(s, \alpha, t)$ bezüglich $\alpha$ stetig, bezüglich $\iota^{\prime}(m+1)$-mal partiell differenzierbar, und diese partiellen Ableitungen, für die wir unter Weglassung der anderen beiden Variablen die Indexschreibweise $g_{r}(t)(r=0,1, \ldots, m+1)$ verwenden, mögèn die Bedingungen

$$
\begin{aligned}
& g_{r}(x)=0 \quad(r=1,2, \ldots, n-1, n+1, \ldots, m-1 \text { mit } m>n \geqq 1) \\
& \text { und } \ddot{g}_{m}(x)>0
\end{aligned}
$$

erfüllen.

1) Bei dieser Arbeit handelt es sich um die •zusammenfassendo Darstellung des ersten Teils der Dissertation B des Autors. 
Im folgénden werden asymptotische Darstellungen dér Funktion $F(s, \alpha)$ für $s \rightarrow s_{0}$ hergeleitet; wobei $s_{0}$ ein Häufungspunkt von $S$ sei (daher wird man sich $S$ von vornherein auf den Durchschnitt mit einer gewissen Umgebung von $s_{0}$ eingeschränkt denkeri). Diese Darstellungen gelten gleichmäßig fiir. alle $\dot{\alpha} \epsilon \cdot A^{\prime}$ bzw. fiir alle $\alpha \in A^{\prime \prime}$, wobei $A^{\prime}, A^{\prime \prime}$ Teilintervalle von $A$ mit $A^{\prime} \cup A^{\prime \prime}=A, A^{\prime} \cap A^{\prime \prime}=\left\{\alpha_{0}\right\}$ sind, die durch die folgenden Eigenschaften von $g(s, \alpha, t)$ charakterisiert werden:

Für alle $(s, \alpha) \in S^{\prime} \times\left(A^{\prime} \backslash\left\{\alpha_{0}\right\}\right)^{\prime}$ möge $g(s, \alpha, t)$ eine einzige stationäre Stelle $y$ mit $\because y>x$ besitzen, es sei $y<b, g_{2}(y)>0$, und $z=y-x$ sei fiir festes $s \in S$ eine streng monotonc Funktion von $\alpha$. Fïr alle $(s, \alpha) \in S \times A^{\prime \prime}$ möge $g(s, \alpha, l)$ kéine stationäre Stelle nit $t>x$ besitzen, und es sei $g_{n}(s, \alpha, x)>0$ für $\alpha \epsilon A^{\prime \prime} \backslash\left\{\alpha_{n}\right\}, g_{n}\left(s, \alpha_{n}, x\right)=0$. Wegen (2) liegt also für $\alpha \in A \backslash\left\{\alpha_{0}\right\}$ in $t=x$ eine stationäre Stelle von $g(s, \alpha, t)$ der Ordnung $n-1$, für $\alpha=\alpha_{0}$ eine dẹ Ordnung $m-1$ vor.

Asymptotische Darstellungen (und Entwicklungen) für $F(s, \alpha)$, die man mit klassischen Methoden, erhalten kann, gelten jeweils nur in 'Teilintervallen von $A^{\prime}$ bzw. $A^{\prime \prime}$, die $\alpha_{0}$ nicht enthalten, oder aber nur für $\alpha=\alpha_{0}$. Die in der vorliegenden Arbeit hergeleiteten gleichmäßigen asymptotischen Darstellungen erfassen auch den Übergang $\alpha \rightarrow \alpha_{0}$ mit $\alpha \in A^{\prime}$ bzw. $\alpha \in A^{\prime \prime}$ (Abschnitte 3 und 4), und in Abschnitt 5 wird sogar eine Darstellung erhalten, die gleichmäßig für alle $a \in A$ gilt. Hiermit werden Resultate aus $[7,8]$ teilweise verbessert und weitgehend verallgemeinert. Zur Herleitung der gleichmäßigen asy'mptotischen Darstellungen, die in [8] nur am Rande untersucht wurden, wird ein Weg beschritten, der sich von den Methoden in ähnlichen Untersuchungen, wie z. B. [3, 2, 9], unterscheidet (Abschnitt 2). In den genannten Arbeiten werden Integrale untersucht, unter denen gégenüber (1) noch der Zusatzfaktor $t^{2} h(t)$ steht, jedoch hat $g(s, \alpha, t)$ die spezicllere Form $s f(\alpha, t)$, und in $[2,8]$ bedeutet $\alpha$ bzw. $-\alpha$ einfach die Lage der Maximalstelle von $f(\alpha, t)$. Ferner beschränken wir uns hier auf die Herleitung asymptotischer Darstellungen; iber asymptotische Entwicklungen von $F(s, \alpha)$ soll in einer gesonderten Veröffentlichung berichtet werden.

\section{Beschreibung der Methode}

Die kritischen Punkte des Integrandén in (1), durch die die Asymptotik von $F(s, \alpha)$ bestimnt wird, sind in Fall $\alpha \in A^{\prime}$ die Maximalstelle $y$ von $-g(t)$ und die untere Integrationsgrenze $\dot{x}$, die für $\alpha=\alpha_{0}$ zusammenfallen. Daher und wegen (2) ziehen wir als Approximante für $F(s, \alpha)$ das Integral

$$
I^{(m, n)}(\tau)=\int_{0}^{\infty} \exp \left(-\Phi^{(m, n)}(\tau, u)\right) d u, \quad \Phi^{(m, n)}(\tau, u)=u^{m}-\frac{m}{n} \tau u^{n},
$$

mit einer gecignet zu wählenden Funktion' $\tau(s, a)$ heran [4]; insbesondere soll $\tau$ für festes $s \in S^{\prime}$ eine streng monotone Funktion von $z^{\prime}$ (und damit von $\dot{\alpha}$ ) sein. Der Integrand in (3) hat im Fall $\tau>0$ genail cin Maximum bei $=\tau^{\frac{1}{m-n}}$. und es gilt $\left.\Phi_{r}^{(m . n)}(\tau, 0)=0^{1}\right)$ für $r=1, \ldots, n-1 ; n+1, \ldots, m-1, \Phi_{m}^{(m, n)}(\tau, 0)>0$, und für $\tau=0$ wird $\Phi_{n}{ }^{(m, n)}(0,0)=0$. Die Integrale (3) sind vom FaxénschènTyp [6]: mit

$$
F i(\alpha, \beta ; x)=\int_{0}^{\infty} \exp \left(-t+x t^{\alpha}\right) t^{\beta-1} d t \quad(0<\alpha<1 ; \beta>0)
$$

?) Indizes bézeichnen wieder partielle Ableitungén bezüglich der letzten Variablèn. 
ist $I^{(m, n)}(\tau)=\frac{1}{m} F i\left(\frac{n}{m}, \frac{1}{m} ; \frac{m}{n}\right)$. Speziell ist $I^{(2,1)}(\tau)=\sqrt{\pi} e^{\gamma^{2}}, \frac{1+\operatorname{erf} \tau}{2}$. Mit der Substitution

$$
t=y+\frac{u-\sigma}{\beta}, \quad \sigma=\tau^{\frac{1}{m-n}}, \quad \beta=\beta(s, \alpha)>0,
$$

bei der über. $\beta$ noch verfiigt wird, ergibt sich

mit

$$
F(s, \alpha)=\frac{1}{\beta} \int_{\sigma-\beta z}^{\sigma+\beta(b-v)} \exp \left(-u^{m}+\frac{m}{n} \tau u^{n}+h^{(m, n)}(s, \alpha, \beta, \tau, u)\right) d u
$$

$$
h^{(m, n)}(s, \alpha, \dot{\beta}, \tau, u)=\Phi^{(m, n)}(\tau, u)-g\left(s, \alpha, y+\frac{u-\sigma}{\beta}\right)
$$

(im folgenden.wird meist kürzer $h(\beta, \tau, u), \Phi(\tau, u)$ und $I(\tau)$ geschrieben). 'Wenn nun' $\beta, \tau$ so gewählt werden, da $\beta \dot{\sigma}+\beta(b-y) \rightarrow \infty$ und $\sigma-\beta z=o$ (1) für $s \rightarrow s_{0}$ und für alle $\alpha \in A^{\prime}$, sowie $h(\beta, \tau, u)=h(\beta, \tau, \dot{\sigma})+o(1)$ gleichmäßig im Integrationsintervall oder wenigstens in einem geeigneten Teilintervall gelten und das Restintegral von geniigend kleiner Ordnung ist, so folgt aus (5) die asymptotische Darstellung

$$
F^{\prime}(s, \alpha) \sim \frac{1}{\beta} \exp (h(\beta, \tau, \sigma)) \int_{0}^{\infty} \exp \left(-\Phi^{(m \cdot n)}(\tau, u)\right) d u \quad\left(s \rightarrow s_{0}, \alpha \in A^{\prime}\right)
$$

Es werden zwei Möglichkeiten der Bestimmung von $\beta ; \tau$ betrachtet, die als besonders zweckmäßig erscheinen. Die Methode ist in etwas modifizierter Form auch im Fall $\alpha \in A^{\prime \prime}$ anwendbar.

\section{Gleichímäßigè asymptotische Darstellungen im Fall $a \in A^{\prime}$}

\subsection{Erste Wahl von $\beta$ und $\dot{\tau}$}

Auf Grund von (4) entsprechen sich die Maximalstellen der Integranden in $F(s, \alpha)$

- nach Ausführung der Substitution (4) - und in $I(\tau)$, und es wird $h_{1}(\beta, \tau, \sigma)=0$. Die Forderung, daß sich auch $t=x$ und $u=0$ entsprechen sollen, führt auf

$$
\sigma=\beta z,
$$

und die weitere Forderung: $h_{2}(\beta, \tau, \sigma)=0$ für $\tau>0$ sowie $h_{m}(\beta, 0,0)=0$, führt auf

$$
\beta=\left(\frac{\cdot g_{2}(y)}{m(m-n) z^{m-2}}\right)^{\frac{1}{m}} \text { für } z>0, \beta=\beta_{0}=\left(\frac{g_{m}(\dot{x})}{m !}\right)^{\frac{1}{m}} \quad \text { für } \quad \dot{z}=0
$$

(es ist $\beta_{0}=\lim _{z \rightarrow 0} \beta$ ). Wegen (8) wird also $\tau=(\beta z)^{m-n}$ mit $\beta$ nach (9), und weiter gilt nuit dieseni $\tau$

$$
\text { - } h(\beta, \tau, \sigma)=-g(y)-\frac{m-n}{n} \sigma^{m}
$$

Zur Herleitung der asymptotischen Darstellung mit diesem Paar $(\beta, \tau)$ betrachten wir statt $F(s, \alpha)$ zunächst das Integral mit gleichem Integranden iiber die Teil- 
intervalle $[x, y+\omega]$ bzw. $\left[y^{\prime}-\omega^{*}, y+\omega^{*}\right]$. Hierin sind $\omega(s), \omega^{*}(s, \alpha)$ Funktionen mit $0<\omega \leqq b-y, 0<\omega^{*} \leqq \min (z, b-y)$.

Satz'1: Es existiere (jeweils für $s \rightarrow s_{0}, \dot{\alpha} \in A^{\prime}$ ) eine Funktion $\omega$ mit

$$
\beta \omega \rightarrow \infty, \quad \beta^{3} \omega^{3} h_{3}(\beta, \tau, \xi)=o(1)^{\prime} \text { für jedes } \xi \in[0,2 \beta \omega] .
$$

Für solche $\alpha$, für die (bei festem $s \in S$ ) $z>\omega$ ausfällt, existiere ein $\omega^{*}$ mit

$\omega^{* 2} g_{2}(y) \rightarrow \infty ; \quad \beta^{3} \omega^{* 3} \Phi_{3}(\tau, \xi)=o(1) \quad$ und $\quad \omega^{* 3} g_{3}(\xi)=o(1)$

für jedes $\xi=\sigma+\vartheta \beta \omega^{*},|\vartheta| \leqq 1$.

Ferner sei $g_{1}\left(\dot{y}+\frac{u-\sigma}{\beta}\right)$ monoton wachsend, wenigstens. für $0 \leqq u \leqq \beta\left(z-\omega^{*}\right)$ und $u>\sigma+\beta \cdot \min \left(\omega, \omega^{*}\right),(s, \alpha) \in \cdot S \times A^{\prime}$. Dann gilt gleichmäßig bezüglich $\alpha \in A^{\prime}$

$$
F(s, \alpha) \sim \frac{1}{\beta} \exp \left(-g(y)-\frac{m-n}{n} \sigma^{m}\right) I^{(m, n)}(\tau), \quad\left(s \rightarrow s_{0}\right)
$$

mit $\beta, \tau$ nach $(8),(\dot{9})$.

Beweis: In den Fällen $z \leqq \omega$ bzw: $z>\omega^{*}$ folgt fiur $F^{(1)}(s, \alpha)=\int_{x}^{y+\omega} e^{-g(t)} d t$ bzw. $F^{(2)}(s, \alpha)=\int_{y-\omega^{*}}^{j+\omega^{\bullet}} e^{-g(t)} d t$ wegen $h_{r}(\beta, \tau, \sigma)=0(r=1,2)$ aus $(11), \operatorname{da} \beta$

$$
F^{(i)}(s, \alpha) \sim \frac{1}{\beta} e^{h(\beta, r, \sigma)} \int e^{-\Phi(r, u)} d u
$$

wobei für $i=1$ die Integrationsgrenzen $0, \beta(z+\omega)$, für $i=2 \beta\left(z-\omega^{*}\right), \beta\left(\ddot{z}+\omega^{*}\right)$ stehen. Wegen $z \leqq \omega$ und $\beta \dot{\omega} \rightarrow \infty$ ist $\dot{\Phi}_{1}(\tau, \beta(z+\omega))=(\beta \omega)^{m-1}$ und weiter

$\int_{\beta(z+\infty)}^{\infty} e^{-\phi(r, u)} d u=o\left((\beta \omega)^{-m+1} e^{-\Phi(x, \beta(z+\omega))}=o\left(\beta \omega e^{-\Phi(r, \beta(z+\omega))}\right)=o\left(\int_{0}^{\beta(z+\infty)} e^{-\Phi(r, u)} d u\right)\right.$,

so daßman für $i=1$ aúf der rechten Seite von (14) die Grenzen $0, \infty$ schreiben darf. Auf Grund der-Monotonie von $g_{1}(t), h(\beta, \tau, \beta(z+\omega))-h(\beta, \tau ; \sigma)=o(1)$ und $h_{1}(\beta, \tau, \beta(z+\omega))=\circ\left(\frac{1}{\beta \omega}\right)($ wegen $(11))$ schließt'man ebenso auf

$$
e^{-h(\beta, z, \sigma)} \int_{\beta(z+\omega)}^{\beta(b-x)} e^{-g\left(y+\frac{u-\sigma}{\beta}\right)} d u=o\left(\int_{0}^{\beta(z+\omega)} e^{-\Phi(r, u)} d u\right)
$$

so da $\beta$ man in (14) $F^{(1)}(s, \alpha)$ durch $F(s, \alpha)$ ersetzen darf. Im zweiten Fall wird $\Phi_{1}\left(\tau, \dot{\beta}\left(z+. \omega^{*}\right)\right) \asymp(\beta z)^{m-2} \dot{\beta} \omega^{*}$, und man kann wie zuvor schließen, nur sind jetzt jeweils zwei Randintegrale abzuschätzen

Für $m=2, \dot{n}=1$ besagen (11) und (12) dasselbe $\left(\omega=, \omega^{*}\right): \omega^{2} g_{2}(y) \rightarrow \infty$, $\omega^{3} g_{3}(\xi)=o(1)$. Aus der ersten der Bedingungen (12) und $\omega^{*} \leqq z$ folgt, daß im zweiten Fall : stets $\tau_{1} \rightarrow \infty$ (oder $z^{2} g_{2}(y) \rightarrow \infty$ ) gilt, ' während für $z \leqq \omega$ die Fälle $\tau=o(1)$, $\tau=1, \tau \rightarrow \infty$ eintreten können. Für $\tau \rightarrow \infty$ ergibt sich aus (13) wegen

$$
\dot{I}^{(m: n)}(\tau) \sim \sqrt{\frac{2 \pi}{m(m-n) \sigma^{m-2}} e^{\frac{m-n}{n} o^{m}}} \quad(\tau \rightarrow \infty)
$$


das für den Fall des inneren Maximums bekannte Resultat

$$
F(s, \alpha) \sim \sqrt{\frac{2 \pi}{g_{2}(y)}} \dot{e}^{-\sigma(y)}
$$

Für $\tau=o(1)$ oder $z^{2} g_{2}(y)=o(1)$ geht (13), wenn noch $z g_{m+1}(\xi)=o\left(g_{m}(y)\right)$ für alle $\xi \in[x, y]$ gilt, in

$$
F(s, \alpha) \sim \frac{1}{m} \Gamma\left(\frac{1}{m}\right)\left(\frac{m !}{g_{m}(x)}\right)^{\frac{1}{m}} e^{-g(x)}
$$

über (speziell gilt (17) im Fall $\alpha=\alpha_{0}$ ). Für $m=2, n=1$ ist die rechte Seite von (17) asymptotisch gleich der Hälfte der rechten Seite von (16).

\subsection{Eine andere Wahl für $\beta$ und $\tau$}

Zu einer weiteren Möglichkeit der Bestimmung von . $\beta$. und $\tau$ gelangt man, indem nächst - analog $\mathrm{zu}[2,3]$ - von der Transformation $-g(s, \alpha, t)=-u^{m}+\frac{m}{n} \tau u^{n}+c$ aúsgegangen und $\tau$ aus der Forderung bestimmt wird, daß sich die kritischen Werte $t=x, t=y$ und $u=0, \dot{u}=\sigma$ entsprechen. So folgt.

$$
\tau=\left[\frac{n}{m-n}\left(g(s, \alpha, x)-g\left(s, \alpha^{\prime}, y\right)\right)\right]^{\frac{m-n}{m}} .
$$

Damit hat die Transformation für uns ihren $Z$ weck erfültt, und wir setzen wie in 3.1 fort, indem wir $\beta$ aus $h_{2}(\beta, \tau, \sigma)=0 \mathrm{zu}$

$$
\beta=\left(\frac{\because-g_{2}(y)}{m(m-n), \sigma^{m-2}}\right)^{\frac{1}{2}} \text { für } \quad \tau>0, \beta=\beta_{0} \quad \text { für } \quad \tau=0 .
$$

bestimmen. Dann wird

$$
h(\beta, \tau, \sigma)^{\prime}=-g(x),
$$

und unter den, Voraussetzungen von Satz 1 ergibt sich, wenn die zweite Bedingung in (11) für $\xi \in[\sigma-\beta z, \sigma+\beta \omega]$ gilt, jetzt

$$
F(s, \alpha) \sim \frac{1}{\beta} e^{-g(x)} I^{(m, n)}(\tau)
$$

mit $\beta, \tau$ nach (18), (19).

\subsection{Hinreichende Bedingungen}

Es werden für den unter 3.1 behandelten Fall Bedingungen angegeben, die hinreichend für die Beziehungen (11) und (12) sind. Dazu benötigen wir einen Hilfssatz.

Hilfssatz: Es möge neben (2) noch

$$
g_{m+1}^{\prime}(\xi)=o\left(g_{m+1}(y)\right) \text {, für jedes } \xi \text { mit } x \leqq \xi \leqq y \quad . \quad(m \geqq 3)
$$

erfüllt sein. Dann lassen sich alle Ableitungen $g_{r}(y)(r \fallingdotseq 2,3, \ldots, m)$ wie folgt durch $g_{2}(y)$ und $g_{m}(y)$ ausdrücken:

$$
g_{r}(y)=\frac{z^{m-r}}{(m-r) !} g_{m}(y)\left[1-\frac{(n-1) !(m-n) !}{(m-1) !}\left(\begin{array}{l}
m-r \\
m-n
\end{array}\right)\right]+O\left(z^{m+1-r} g_{m+1}(y)\right),
$$


$g_{r}(y) \doteq \frac{(r-1) !}{m-n} d_{r} \frac{g_{2}(y)}{z^{r-2}}+O\left(z^{m+i-r} g_{m+1}(y)\right) \quad$ mit $\quad d_{r}=\left(\begin{array}{c}m-1 \\ r-1\end{array}\right)-\left(\begin{array}{l}n-1 \\ r-1\end{array}\right)$.

Beweis: Mit dér Taylor-Entwicklung von $g_{r}(x)(n+1 \leqq r \leqq m-1)$ um $t=y$ läßt sich wegen (2) $g_{r}(y)$ ausdrücken, und man erhält (23) zunächst für $r=m-1$ und dann für alle vorhergehenden $r$, indem man die jeweils zuvor erhaltenen Resultate einsetzt, so daß (23) im Fall $n=1$ bewiesen ist. Für $n>1$ folgt aus $g_{1}(y)$ $\doteq \frac{z^{n-1} g_{n}(x)}{(n-1) !}+\frac{z^{m-1} g_{m}(x)}{(m-1) !}+O\left(z^{m} g_{m+1}(y)\right)=0$ und $g_{m}(x)=g_{m}(y)+O\left(z g_{m+1}(y)\right)$, da $\beta$

$$
g_{n}(x)=-\frac{(n-1) !}{(m-1) !} z^{m-n} g_{m}(y)+O\left(z^{m-n+1} g_{m+1}(y)\right)^{-}
$$

gilt. Vergleicht man (25) mit der Taylor-Entwicklung von $g_{n}(x)$ um $t=y$, ergibt sich (23) für $r=n$, und, indem man wieder wie oben vorgeht, für alle genannten $r$ : Wènn man (23) für $r=2$ nach $g_{m}(y)$ auflöst und.für $r=3, \ldots, m \div 1$ in (23) einsetzt, ergibt sich (24)

Wenn außer den Voraussetzungen des Hilfssatzes noch

$$
\delta=\frac{z g_{m+1}(y)}{g_{m}(y)}=o(1)
$$

erfüllt ist, kann man (23) und (24) als asymptotische Gleichungen schreiben. Aus (2), und (25) folgt dann $g_{n}(x)<0$ für $z>0$.

Es seien die Voraussetzungen des Hilfssatzes erfiillt. Dann kann, wenn (26) gilt, die erstè der Bedingungen (11) in der Form

$$
\omega^{m} g_{m}(y) \rightarrow \infty
$$

geschrieben werden, da $(\beta \omega)^{m}=\frac{\omega^{m} g_{2}(y)}{z^{m-2}}=\omega^{m} g_{m}(y)$ wegen (9) und (24) ist. Wegen $h_{f}(\beta, \tau, \sigma)=m(\dot{r}-1) ! d_{r} \sigma^{m-r}-\frac{1}{\beta^{r}} g_{r}(y)(r=3, \ldots, m)$ folgt für diese $r$ :

$$
h_{r}(\beta, \tau, \sigma)=O\left({ }_{\delta}\left(z g_{m}^{\frac{i}{m}}(y)\right)^{m-r}\right)=O\left(\delta \sigma^{m-r}\right)^{\prime} .
$$

Also ist für $z \leqq \omega$, wenn (22) sogar für das Intervall $x \leqq \xi \leqq y+\omega$ gilt,

$$
\omega^{3} \beta^{3} \grave{h}_{3}^{\prime}(\xi)=O\left(\delta(\beta \omega)^{m}\right)=O\left(\omega^{m+1} g_{m+1}^{\prime}(y)\right)
$$

und daher $\omega^{m+1} g_{m+1}(y)=o(1)$ hinreićhend für die zweite der Bedingungen (11). Indem man (22) hinzu nimmt, erhält man neben $(27)$ noch

$$
\omega^{m+1} g_{m+1}(\xi)=o(1) \cdot(x \leqq \xi \leqq y+\omega)
$$

als hinreichende Bedingungen für (11) (für $z \coprod^{-} \omega$ folgt aus (27), (28) auch (26)). Für $m=2$ sind diese mit (11) identisch. Beachtet man in $(12) \Phi_{3}(\tau, \xi)=O\left(\sigma^{m-3}\right)$ (Taylor-Entwicklung!), so daß $\beta^{3} \omega^{* 3}, \Phi_{3}(\tau, \xi)=O\left(\omega^{* 3} \beta^{m} z^{m-3}\right)=O\left(\omega^{* 3} \frac{g_{2}(y)}{z}\right)$ wird, erhält man mit

$$
\omega^{* 3} g_{2}(y)=o(z)
$$

eine hinreichende Bedingung für die zweite der Rélationen (12) (vgl. [1] für $m=3$ ): 
Aus dem Hilfssatz folgt noch: für $\tau, \beta$ entsprechend (18), (19) gilt wegen (23) und (24):

$$
\begin{aligned}
& \sigma^{m}=\tau^{\frac{m}{m-n}}=\frac{z^{2} g_{2}(y)}{m(m-n)}+O\left(z^{m+1} g_{m+1}(y)\right)=\frac{z^{m} g_{m}(y)}{m !}(1+O(\delta)), \\
& \beta=\left(\frac{g_{2}(y)}{m(m-n) z^{m-2}}\right)^{\frac{1}{m}}(1+O(\delta))=\left(\frac{g_{m}(x)}{m !}\right)^{\frac{1}{m}}(1+O(\delta))
\end{aligned}
$$

so daß in Fall $\delta=o(1)$ die Funktionen $\tau, \beta$ asymptotisch gleich denen nach (8), (9) sind. Für den unter 3.2 behandelten Fall lassen sich daher entsprechende hinreichende Bedingungen wie oben formulieren.

\section{GleichmäBige asymptotische Darstellungen im Fall $a \in A^{\prime \prime}$}

\subsection{Erste Wahl von $\beta$ und $\tau$}

Zur Herleitung asymptotischer Dąrstellungen im Fall $\alpha \in A^{\prime \prime}$ wird als Approximante wieder das Integral' (3) verwendet, jetzt aber mit $\tau \leqq 0$ ( $\tau$ soll für festes $\dot{s} \in S$ eine streng monotone Funktion von $\alpha$ sein). Statt (4) wird

$$
t=x+\frac{u}{\beta}, \quad \beta=\beta(s, \alpha)>0
$$

substituiert, was in (6) zu einer Ānderung im Argument von $g$ führt. Wegen (2) ist dann $h_{r}(\beta, \tau, 0)=0$ für $r^{-}=1, \ldots, n-1, n+.1, \ldots, m-1$. Fordert man außerdem $h_{n}(\beta, \tau, 0)=h_{m}(\beta, \tau, 0)=0$, so ergeben sich für $\beta$ und $\tau$ :

$$
\beta=\left(\frac{g_{m}(x)}{m !}\right)^{\frac{1}{m}}, \quad \tau=-\frac{g_{n}(x)}{m(n-1) ! \beta^{n}}
$$

Es gilt nun folgende Aussage.

Satz 2: Es sei $g_{m+1}(x) \neq 0$, und es existiere eine Funktion $\omega=\omega(s, \alpha)$. mit $0<\omega \leqq b-x$, die den Bedingungen

$$
\beta \omega \rightarrow \infty, \omega^{m+1} g_{m+1}(x)=o(1), g_{m+1}(\xi) \doteq O\left(g_{m+1}(x)\right) \quad \text { für alle } \xi \in[x, x+\omega]
$$

(jeweils für $s \rightarrow s_{0}$ und für jedes ' $\alpha \in A^{\prime \prime}$ ) genügt. Ferner sei $g_{1}(t)$ monoton wachsend für $x+\omega<t<b$. Dann gilt für $s \rightarrow s_{0}$ und gleichmäßig für alle $\alpha \in A^{\prime \prime}$ :

$$
F(s, \alpha) \sim \frac{1}{\beta} e^{-g(x)} I^{(m, n)}(\tau)
$$

mit $\beta, \tau$ nach (33).

Beweis: Man erhält die Behauptung zunächst für das Integral $F^{(3)}(s, \dot{\alpha})$ $=\int_{x}^{x+\omega} e^{-g(t)} d t$,-indem der Integrand wie in (5) dargestellt, $h(\beta, \tau, u)$ in $u=0$ nach Taylor bis zum Restglied $m$-ter Ordnung entwickelt sowie (34) und $h(\beta, \tau, 0)=-g(x)$. beachtet wird. Ferner ist

$$
\int_{\beta \omega}^{\infty} e^{-\phi(t, u)} d u=O\left(\frac{e^{-\phi(\tau, \beta \omega)}}{\phi_{1}(\tau, \beta \omega)}\right)=O\left(\beta \omega e^{-\phi(r, \beta \omega)}\right)=o\left(\int_{0}^{\beta \omega} e^{-\phi(r, u)} d u\right)
$$



und wie im Beweis zu Satz 1 zeigt man, daß $\int_{x+\omega}^{0} e^{-g(t)} d t$ eine kleinere Ordnung als die
rechte Seite von (35) hat

. Wenn $g_{m+k}(x)=0(k=1, \ldots, l-1)$ gilt, ist in (34) der Index $m+1$. durch $m+l$ zu ersetzen. Für $\tau=o(1)$ folgt aus (35) wieder (17), für $\tau \rightarrow-\infty$

$$
F(s, \alpha) \sim \frac{1}{n} \Gamma\left(\frac{1}{n}\right)\left(\frac{n !}{g_{n}(x)}\right)^{\frac{1}{n}} e^{-g(x)} .
$$

Nach (31) unterscheiden sich die durch (9), (19), (33) definierten Funktionen $\beta(s, \alpha)$ un' den Faktor $1+O(\delta)$, und nach (25) (30) gilt entsprechendes für die durch (8), (18) und (33) definierten Funktionen $\tau$, so daß alle bisher verwendeten $\beta, \tau$ jeweils, unter sich asymptotisch gleich sind, 'solange $\delta=o(1)$ erfüllt ist. Unter $y$ soll dabei im Fall $\dot{\alpha} \in A^{\prime \prime}(\tau \leqq 0)$ ein Sattelpunkt von $g(t)$ verstanden werden, der für $\alpha \rightarrow \alpha_{0}$ gegen $x$ strebt (insbesondere kann $y$ eine Maximalstelle von $-g(t)$ sein, die für $\alpha \neq \alpha_{0}$ außerhalb des. Integrationsintervalls liegt), und die benötigten partiellen Ableitungen von $g(t)$ sollen existieren. Es werde nun $\omega$ so gewählt, daß (27) und (28) (mit $x \leqq \xi \leqq y+\omega$, wenn $\alpha \in A^{\prime}$, und mit $\dot{x} \leqq \xi \leqq x+\omega$; wenn $\alpha \in A^{\prime \prime}$ ) gelten. Das hat zur Folge, daß die Voraussetzungen (11), (34) aús den Sätzen 1 und 2 erfüllt sind. Ferner sei $|z| \leqq \dot{\omega}$. Dann läßt sich zeigen, daß $\exp \left(-g(y)+h\left(\beta, \beta z,(\beta z)^{m-n}\right)\right)$ $\sim \exp (-g(x))-$ mit $\beta$ nach $(9)^{\prime}-$ ist und ferner die $I^{(m, n)}(\tau)$ für die drei verschiedenen $\tau$ unter sich gleich sind. Daher sind dann die asymptotischen Darstellungen (13), (21) und (35) unter sich asymptotisch gleich.

\subsection{Zweite Wahl von $\dot{\beta}$ und $\tau$,}

In Verallgemeinerung zu (18) werde jetzt

$$
\tau=\operatorname{sgn}\left(g_{n}(x)\right)\left[\frac{n}{m-n} \cdot g(x)-g(y) \mid\right]^{\frac{m-n}{m}}
$$

gewählt, wobei $y$ dic am Ende von 4.1 genannte Bedeutung hat (eine Verallgemeinerung von (18) liegt tatsächlich vor, wenn $g_{n}(x)<0$ für $z>0$ gilt, was angenommen werde -. vgl. dazu die Bemerkung in Anschlu $B$ an (26)). Das.zugehöríge $\beta$ werde aus $h_{n}(\beta, \tau, 0)=0$ bestimmit:

$$
\beta=\left(\frac{-g_{n}(x)}{m(n \dot{-1}) ! \tau}\right)^{\frac{1}{n}}
$$

Wir setzen voraus, da $\beta \tau$ und $\beta$ den Beziehungen (30), (31) mit $|z| \leqq \omega$ genügen, woraus wegen $\beta>0$ 'folgt,. da $B \arg z=\frac{1}{m-n} \arg \tau=\frac{\pi+2 k \pi}{m-n}(k$ ganz $)$ sein muß. Dann gilt ein zu Satz 2 entsprechender Satz mit $\tau, \beta$ nach (37), (38). Der Beweis verläuft wie dort; zusätzlich ist $(\beta \omega)^{m} h_{m}(\beta, \tau, 0)=o\left(\omega^{m+1} g_{m+1}(x)\right)$ zu beachten.

\section{Eine gleichmäBige asymptotische Darstellung für $a \in A$}

Nach der Bemerkung am Ende v'on 4.1 gilt unter den dort genannten und den restlichen Voraussetzungen aus Satz 1 die asymptotische Darstellung (21) mit $\tau$ nach (18) ,und $\rho^{\prime}$ nach (19) auch noch für solche $\alpha \in A^{\prime \prime}$, für die $|z|=|y-x| \leqq \omega$ ist. Wenn aber $-\tau$ hinreichend stark gegen $\infty$ strebt, gibt (21) das asymptotische Ver- 
halten von $F(s, \alpha)$, das dann durch (36) bestimmt ist, nicht mehr richtig wieder. Um auch diesen Fall zu erfassen, addieren wir zur rechten Seite von (21) die Differenz. aus der rechten Seite von (36) und der sich aus (21) für $\tau \rightarrow-\infty$ ergebenden Darstellung. Nun erhalten wir eine asymptotische Darstellung für $F(s, \alpha)$, dic den zuvor nicht erfaßten Fall überdeckt und unter der Zusatzvoraussetzung $\delta^{n}=o(\tau)$ für alle $\alpha \in A$ gleichmäßig gültig ist:

$F(s, \alpha) \sim e^{-g(x)}\left\{\frac{1}{\beta^{+}} \int_{0}^{\infty} \exp \left(-u^{m}+\frac{m}{n} \tau u^{n}\right) d u+\frac{1}{n} \Gamma\left(\frac{1}{n}\right)\left(\frac{n}{-m \tau}\right)^{\frac{1}{n}} \cdot\left(\frac{1}{\beta^{-}}-\frac{1}{\beta^{+}}\right)\right\}$

Hierin bezeichnèn $\beta^{-}, \beta^{+}$die Funktionen $\beta$ nach (38) bzw. (19) (es sèi angenommen, $\operatorname{da} \beta \beta^{+}$für alle $\tau<0$ reell ausfällt). Für $|z| \leqq \omega$ hat nämlich das Zusatzglied eine kleinere Ordnung als der erste Summand, 'und für $\alpha \in A^{\prime}, z \geqq \omega$ trifft das für beide Summanden des Zusatzgliedes einzeln zu. Bei der unvollständigen Gamma-Funktion $\Gamma(s-+1, a)$ führt (39) auf das in $[8,(56)]$ angegebene Resultat.

\section{Ein Beispiel}

Wir betrachten als Beispiel die zur Anger-Funktion in Beziehung stehende Funktion

$$
A_{-8}(\alpha s)=\int_{0}^{\infty} \exp (-s(\alpha \cdot \sinh t-t)) d t \quad(\alpha>0)
$$

Aus der Literatur. sind mehrere, mit verschiedenen Methoden hergeleitete gleichmäßige asymptotische Darstellungen für $s \rightarrow \infty$ bekannt (vgl. $[1,4,6])$. Diẹse und noch weitere lassen sich auch mit unserer einheitlichen Methode erhalten: Es ist $A^{\prime}=(0,1], A^{\prime \prime}=[1, \infty)$ und $\alpha_{0}=1$.

Für $\alpha \in A^{\prime}$ wird, wenn $\alpha=\frac{1}{\cosh \gamma}$ eingeführt wird, $y=z=\gamma$. Da $g_{2}(0)=0$, $\operatorname{gr}(0)=0$ für $r=1,3$ ist, liegt der. Fall $m=3, n=1$ vor. Nach 3.1 wird $\beta=\left(\frac{s \tanh \gamma}{6 \gamma}\right)^{\frac{1}{3}}$ für $\gamma>0, \beta_{0}=\left(\frac{s}{6}\right)^{\frac{1}{3}}, i=\left(\frac{s \gamma^{2} \tanh \gamma}{6}\right)^{\frac{2 j}{3}}$ und somit, da die Voraussetzungen von Satz 1 erfüllt sind;

$$
\underset{\left(s \rightarrow \infty, \alpha \in A^{\prime}\right)}{A_{-8}(\alpha s) \sim \frac{1}{\beta} \exp \left(s\left(\gamma-\tanh \gamma-\frac{1}{3} \gamma^{2} \tanh \gamma\right)\right) \int_{0}^{\infty} \exp \left(-u^{3}+3 \tau u\right) d u}
$$

(vgl. [1]): Entsprechend wird nach $3.2 \tau=\left[\frac{s}{2}(\gamma-\tanh \gamma)\right]_{\gamma}^{\frac{2}{3}}$,

$\beta=\left(\frac{s}{6}\right)^{\frac{1}{3}} \cdot \frac{(\tanh \gamma)^{\frac{1}{2}}}{3(\gamma-\tanh \gamma)^{\frac{1}{6}}}$ für $\gamma \neq 0$ und $\beta=\beta_{0}$ für $\gamma=0$. Mit diesen $\tau, \beta$ gilt

$$
\dot{A}_{-\dot{s}}(\alpha s) \sim \frac{1}{\beta} I^{(3,1)}(\dot{\tau}) \quad \cdot \quad\left(s \rightarrow \infty, \alpha \in A^{\prime}\right)
$$

(die rechte Seite ist das Fïhrungsglied der bei OLver [6] angegebenen asymptotischen Entwicklung). 
Für $\alpha \geqq 1$ folgt mit, Satz 2

$$
A_{-s}(\alpha s) \sim\left(\frac{6}{\alpha s}\right)^{\frac{1}{3}} \int_{0}^{\infty} \exp \left(-u^{3}-\left(\frac{6 s^{2}}{\alpha}\right)^{\frac{1}{3}}(\alpha-1) u\right) d u \quad\left(s \rightarrow \infty, \alpha \in A^{\prime \prime}\right)
$$

(vgl. [4]). Nach 4.2 wird $\left(\right.$ mit $\left.y=i, \arccos \frac{1}{\alpha}\right)$ für $s \rightarrow \infty, \alpha \in A^{\prime \prime}$

$$
A_{-8}(\alpha s) \sim \frac{-3 \tau}{s(\alpha-1)} I^{(3,1)}(\tau), \quad \tau=-\left[\frac{s}{2}\left(\sqrt{\alpha^{2}-1}-\arccos \frac{1}{\alpha}\right)\right]^{\frac{2}{3}}
$$
erhalten. Schließlich folgt mit $\tau=\operatorname{sgn}(1-\alpha)\left[\frac{s}{2}\left|\operatorname{arcosh} \frac{1}{\alpha}-\sqrt{1-\alpha^{2}}\right|\right]^{\frac{2}{3}}$ (hierin
sind die $\tau$ aus (42) und. (44) enthalten),

$$
\begin{gathered}
\beta^{-}=-\frac{s(\alpha-1)}{3 \tau}, \beta^{+}=\left(\frac{s}{6} \sqrt{\left.\frac{1-\alpha^{2}}{\tau}\right)^{\frac{1}{2}}} \text { für. } s \rightarrow \infty, \alpha>0\right. \\
A_{-s}(\alpha s) \sim\left(\frac{6}{s}\right)^{\frac{1}{2}}\left(\frac{\tau}{1-\alpha^{2}}\right)^{\frac{1}{4}} \int_{0}^{\infty} \exp \left(-u^{3}+3 \tau u\right) d u+\frac{1}{s(\alpha-1)} \\
\quad+\frac{1}{3 \tau}\left(\frac{6}{s} \sqrt{\frac{\tau}{1-\alpha^{2}}}\right)^{\frac{1}{2}} .
\end{gathered}
$$

\begin{tabular}{|c|c|c|c|c|}
\hline$a$ & genauer Wert & Wert nach (41) & Wert nach (42) & Wert nach (45) \\
\hline $\begin{array}{l}0,4 \\
0,5 \\
0,6 \% \\
0,7 \\
0,8 \\
0,9 \\
0,95\end{array}$ & $\begin{array}{c}560,07 \\
78,735 \\
17,847 \\
5,7434 \\
2,4133 \\
1,2419 \\
0,94560\end{array}$ & $\begin{array}{c}557,78 \\
78,506 \\
17,842 \\
5,7657 \\
2,4330 \\
1,2555 \\
0,95677\end{array}$ & $\begin{array}{c}558,62 \\
78,585 \\
17,831 \\
5,7485 \\
2,4210 \\
1,2517 \\
0,95532\end{array}$ & $\begin{array}{c}558,60 \\
78,570 \\
17,817 \\
5,7359 \\
2,4094 \\
1,2410 \\
-0,94498\end{array}$ \\
\hline$\alpha$ & genauer Wert & Wert,nach (43) & Wert nach (44) & Wert nach (45) \\
\hline $\begin{array}{l}1 \\
1,05 \\
1,1 \\
1,5 \\
2 \\
4\end{array}$ & $\begin{array}{l}0,74360 \\
0,60111 \\
0,49751 \\
0,18561 \\
0,098268 \\
0,033285\end{array}$ & $\begin{array}{l}0,75317 \\
0,60676 \\
0,50094 \\
0,18575 \\
0,098279 \\
0,033285\end{array}$ & $\begin{array}{l}0,75317 \\
0,60754 \\
0,50195 \\
0,18609 \\
0,098358 \\
0,03.3289\end{array}$ & $\begin{array}{l}0,74317 \\
0,60080 \\
0,49729 \\
0,18557 \\
0,098260 \\
0,033284\end{array}$ \\
\hline
\end{tabular}

In der folgenden Tabelle sind fiir $s=10$ und einige $\alpha$ die mit (41) bis (45) erhaltenen Werte den genauen Funktionswerten gegenübergestellt:

\section{LITERATUR}

[1] Berg, L.: Asymptotische Darstellungen und Entwicklungen. Berlin 1968.

[2] Bleistein, N.: Uniform asymptotic expansions of integrals with stationary point near algebraic singularity. Comm: Pure Appl: Math. 19 (1966), 353-.370.

[3] Chester, C., Friedman, B., and F. Ursell: An extension of the method of steepest descents. Proc. Camb. Phil. Soc. 53 (1957), 599-611. 
[4] Erdélyi, A.: Uniform asymptotic expanșions of integrals. In: Analytic methods in mathematical physics (Editors: Gilbert, R. P., and R. G. Newton), 149-168. New York 1970.

[5] Erdélyi, A., and M. Wyman: The asymptotic evaluation of certain integrals. Arch. Rat. Mech. Anal. $14(1963), 149-168$.

[6] Orver, F. W. J.: Asymptotics and special functions. New York-London 1974.

[7] Scheid, H.-J.: Asymptotische Darstellungen für Parameterintegrale mit zwei reellen Parametern I. Math. Nachr. S2 (1978), 309-323.

[8] Scheld, H.'-J.: II. Math. Nachr. 88 (1979), 391-408.

[9] Woxg, R.: On uniform asymptotic expansion of definite integrals. J. Appr. Th. 7 (1973), $76-86$.

' Manuskripteingang: 25. 08. 1981

V́ERFASSER:

Doz..Dr. sc. Hans-Joachim SçHell

Sektion Mathematik der Technischen Hochschule

DDR-9010 Karl-Marx-Stadt, Str. der Nationen 62 